\title{
Paediatric aspects: no fasting in children?
}

\author{
Staffan Meurling
}

Department of Paediatric Surgery, University Children's Hospital, Uppsala, Sweden

\section{Abstract}

Fasting overnight is not necessary in elective surgery for children and will probably cause metabolic complications. Two hours or less of fasting is the optimal time in most paediatric patients, irrespective of age.

Fasting before general anaesthesia aims to minimize the risk of aspiration by reducing the volume and acidity of stomach contents.

The guidelines for adults have until recently been nil by mouth from midnight before the operation. In the present issue of SJN Olle Ljungqvist reviews the concept of preoperative oral carbohydrate treatment in adults. A few randomized studies, mostly based on healthy adults, compared nil by mouth with allowing clear fluid (water, coffee, tea, fruit juice, etc.) $2 \mathrm{~h}$ preoperatively. They found no evidence of larger volume or lower $\mathrm{pH}$ of the gastric contents when fluid was allowed $2 \mathrm{~h}$ before surgery. On the contrary, many studies showed significantly lower gastric volumes. To my knowledge no such studies have been performed in children. Neither have metabolic studies been conducted in children to compare preoperative fasting and non-fasting. Paediatric surgery, and especially neonatal surgery, aims for minimal preoperative metabolic disturbance, but must still ensure the safety of the anaesthesia. For that reason most paediatric anaesthesiologists allow children attending for elective surgery clear fluids even later than $2 \mathrm{~h}$ before surgery - until the child leaves the ward for the operation theatre. The child is then anaesthetized under optimal conditions of hydration. The cost of intravenous fluid is also omitted. Infants (elective surgery) who still are being breast-fed at $2-3 \mathrm{~h}$ intervals do not need to fast, but one should be aware that breast milk contains a substantial amount of fat which prolongs the emptying time of the stomach.

If infants and children are given clear fluid until they leave the ward, they seem to be happier and easier to handle.

It is important to avoid long-lasting fasting in all age groups since fasting can, especially in the lower age group, result in serious metabolic disturbances, such as hypoglycaemia and ketonaemia. Furthermore, infants have a low energy reserve.

There are no hard facts to support the need for children attending for elective surgery, irrespective of age, to fast for longer than $2 \mathrm{~h}$. It may be an advantage to let children have clear fluid until they leave the ward.

Staffan Meurling

Department of Paediatric Surgery

University Children's Hospital

SE-75I 85 Uppsala

Sweden

E-mail: staffan.meurling@surgsci.uu.se 